\title{
Verfassungsrechtliche Schuldenbremsen im Föderalismus
}

\author{
Roland Sturm
}

Die Begrenzung der Staatsverschuldung ist kein Föderalismusthema. Dieser Zusammenhang wird in erster Linie dadurch hergestellt, dass internationale Organisationen, beispielsweise die Europäische Union, die gesamtstaatliche Verschuldung politisch und ökonomisch bewerten. So wird das Augenmerk darauf gelenkt, dass die nationale Staatsverschuldung mehr ist als die Verschuldung des Bundes. In Deutschland umfasst sie auch die Schulden der Länder, der Kommunen und der Sozialversicherungen. Für Bundesstaaten stellt nicht nur die Schuldenbegrenzung an sich ein Problem dar, sondern auch die innerstaatliche Umsetzung von Verschuldungsregeln. Der zentralstaatliche Durchgriff sollte hier ersetzt werden durch ein föderalismusschonendes Arrangement, das nicht mit Hilfe hierarchischer Steuerung, sondern auch durch Anreize, Verhandeln und Überzeugen dem Ziel eines ausgeglichenen Haushalts ohne Neuverschuldung näher kommt. Je unitarischer der Föderalismus ist, desto leichter fallen in diesem Zusammenhang top-down Verfahren und desto weniger wird die gliedstaatliche Präferenzbildung beziehungsweise die Zustimmung der Länder zu bestimmten Haushaltsregeln berücksichtigt.

Anregungen für einen Mechanismus zur Begrenzung der Staatsverschuldung hat sich die deutsche Politik bei den Vorgaben des Stabilitäts- und Wachstumspaktes der EU und in der Schweiz geholt, diese aber nicht vollständig übernommen. Die langjährige positive Erfahrung föderaler angelsächsischer Länder wie Kanada oder USA, aber auch des Vereinigten Königreichs, mit „balanced budget“-Regeln wurde in der deutschen Diskussion weitgehend ignoriert. ${ }^{1}$ Das Vereinigte Königreich verdient Erwähnung, selbst wenn es sich hier nicht um einen föderalen Staat handelt, denn die hier zeitweise gewählte erfolgreiche Strategie zum Erreichen eines ausgeglichenen Haushalts und zum Abbau der Staatsverschuldung ließe sich ohne weiteres auch auf deutsche Verhältnisse übertragen.

Hier sollen nur zwei in den USA beziehungsweise in Großbritannien erfolgreiche „Schuldenbremsen“ kurz erwähnt und im Folgenden erläutert werden, die „pay-as-you-go“ (PAYGO)-Regel und das System der „cash limits“. ${ }^{2}$ Beide Haushaltsregeln könnten zwar

1 Vgl. Roland Sturm, Mechanismen der Defizitbegrenzung im internationalen Vergleich, in: Ralf Thomas Baus / Annegret Eppler / Ole Wintermann (Hrsg.), Zur Reform der föderalen Finanzverfassung in Deutschland, Baden-Baden 2008, S. 237 - 243. Siehe auch bereits die Überlegungen und rechtsvergleichenden Ausblicke von Wolfgang Göke als seinerzeitiges Mitglied des Niedersächsischen Landesrechnungshofs: ders., Staatsverschuldung - Finanzverfassungsrechtliche Ursachen und Lösungen zu ihrer Begrenzung, in: Niedersächsische Verwaltungsblätter (NdsVBl.), 3. Jg. (1996), H. 1, S. 1 ff.; ders., Auf dem Weg zur Konsolidierung der Landesfinanzen? Anmerkungen zu den Landeshaushalten 2004-2006, in: NdsVBl., 13. Jg. (2006), H. 5, S. 125 ff.; ders., Staatsverschuldung - Zur desolaten Lage des Finanzverfassungsrechts des Bundes und der Länder, in: Zeitschrift für Gesetzgebung, 21. Jg. (2006), H. 1, S. 1 - 27.

2 Hierzu und zu weiteren Haushaltsregeln vgl. Roland Sturm, Haushaltspolitik in westlichen Demokratien. Ein Vergleich des haushaltspolitischen Entscheidungsprozesses in der Bundesrepublik Deutschland, Frankreich, Großbritannien, Kanada und den USA, Baden-Baden 1989. 
auf Bundes- wie auf Landesebene Anwendung finden, haben aber in Deutschland auf absehbare Zeit aus zwei Gründen keine Chance: Erstens fordern sie von den politisch Verantwortlichen die Fähigkeit und Bereitschaft zu weitgehend unpopulären tiefen finanziellen Einschnitten auf einer Reihe von Politikfeldern, und zweitens wäre ein geradezu revolutionärer „Kulturwandel“ in der deutschen Haushaltspolitik erforderlich, der das Denken in Ausgaben mit politisch verhandelbaren Grenzen durch ein Denken in einem klar begrenzten Einnahmenrahmen ohne eine Ausgaben ermöglichende „politische Hintertür“ ersetzt.

Die deutsche Politik scheint zuversichtlich, dass sie mit dem wichtigsten Ergebnis der „Föderalismusreform II“ von $2009^{3}$, der „Schuldenbremse“, ein effizientes Instrument der Haushaltspolitik gefunden hat. Sie empfiehlt es föderalen wie nichtföderalen Staaten der Europäischen Union zur Nachahmung. Finanzminister Wolfgang Schäuble sagte am 9. Juni 2010 auf einer Veranstaltung des CDU-Wirtschaftsrats: „Ohne Schuldenbremse im Grundgesetz hätte kein Politiker in diesen Zeiten das Amt des Bundesfinanzministers antreten können.“ 4

\section{Schuldenbremsen in Großbritannien, den USA und der Schweiz}

Am Ende der Regierungszeit Margaret Thatchers (1979 bis 1990) gelang es in Großbritannien nicht nur, den Haushalt auszugleichen, sondern sogar noch Überschüsse zu erwirtschaften und den Anteil der Staatsverschuldung am Bruttoinlandsprodukt deutlich zu reduzieren. Hierzu trug unter anderem die Orientierung der Ausgabenpolitik an der Einnahmensituation bei, die haushaltstechnisch 1982 vollzogen wurde. Das neue System der cash limits zog für den Haushalt eine Ausgabenobergrenze ein, die auch durch die Inflationsentwicklung unbeeinflusst blieb. Damit verlor der Ausgabenbedarf jegliche normative Kraft für die Haushaltsentscheidung. Das Definieren einer einnahmenorientierten Ausgabenobergrenze hatte für die politischen Akteure den Effekt, dass sie - wie dies Margaret Thatcher als Premierministerin auch tat - Verantwortung für die Prioritätensetzungen in der Ausgabenpolitik übernehmen mussten. Dies bedeutete, zahlreichen Ausgabenwünschen zu widerstehen, auf wahlkampfwirksame Ausgaben zu verzichten und Ausgabenkürzungen in Parlament und Öffentlichkeit zu vertreten. Der Ausweg in die Staatsverschuldung stand nicht zur Verfügung. Für Politiker bedeutet das System der cash limits: Aus „spenders“ sollen effizienzorientierte „manager“ öffentlicher Ausgaben werden.

Die PAYGO-Regel wurde in den USA 1990 vom Kongress durch den Budget Enforcement Act (BEA) sowie 1997 durch den diesen weiter konkretisierenden Balanced Budget Act zur Grundlage der amerikanischen Defizitbegrenzungspolitik. Im Rahmen der PAYGO-Regel werden jährliche Grenzen für nichtobligatorische Ausgaben definiert und durchgesetzt. Die Regel verlangt eine Kompensation für neue Ausgaben durch höhere Steuern oder das Kürzen anderer Ausgaben. Werden die Ausgabengrenzen nicht eingehalten oder wird die PAYGO-Regel verletzt, zieht dies automatisch Kürzungen für alle Ausgaben „nach der Rasenmähermethode“ nach sich. Die PAYGO-Regel bewährte sich in der Praxis als effiziente Methode des Haushaltsausgleichs. Ende der 1990er Jahre wies der amerikanische

3 Ausführlicher ders., Föderalismus, Baden-Baden 2010.

4 Zitiert in Manfred Schäfers, Die Bremse für den Finanzminister, in: FAZ vom 10. Juni 2010, S. 13. 
Bundeshaushalt Überschüsse auf. In dieser Situation und angesichts von Projektionen weiter wachsender Haushaltsüberschüsse war es politisch nicht mehr möglich, die Haushaltsdisziplin im Kongress aufrecht zu erhalten. Die jährlichen Ausgabenbegrenzungen wurden erweitert, und der BEA wurde durch „Notausgaben“ („emergency spending“), für die der BEA nicht galt, unterlaufen. 2002 wurde der BEA nicht mehr erneuert. Ohne die PAYGORegel entstanden erneut defizitfinanzierte Haushalte, wie die OECD jüngst bekräftigte. ${ }^{5}$

In der Schweiz gab es bereits vor der Einführung einer Bundesschuldenbremse auf kantonaler Ebene Haushaltsregeln mit deutlicher Bremswirkung für die Staatsverschuldung, wie Lars P. Feld ausführt: „Die erfolgreichen kantonalen Schuldenbremsen zwingen die Parlamente zu Anpassungen auf der Einnahmenseite. Da die Ausgaben häufig bereits durch ein Finanzreferendum restringiert sind und Steuersenkungen im Steuerwettbewerb eine große Versuchung für die Parlamentarier darstellen, ist diese institutionelle Ausgestaltung für die Schweizer Kantone gut geeignet. "6 Elf der 26 Kantone haben seit den 1990er Jahren Schuldenbremsen eingeführt, die anderen zahlen höhere Risikoaufschläge für ihre Verschuldung, was den Anreiz zur Neuverschuldung ebenfalls dämpft. Charles B. Blankart und Erik $R$. Fasten sehen die entscheidende Voraussetzung für die Beachtung der selbst gesetzten Schuldenbremsen der Kantone in der Freiwilligkeit. Top-down-Modelle - im Sinne zentralstaatlicher Vorgaben - haben Probleme der Legitimation und ein Folgebreitschaft-Problem. ${ }^{7}$

Auf der Bundesebene hat man in der Schweiz längere Zeit eine „Schuldenbremse“ diskutiert, 2001 beschlossen und 2003 schließlich in Kraft gesetzt. Diese strebt über den Konjunkturzyklus hinweg einen ausgeglichenen Haushalt an. Eine Notfallregel lässt finanzielle Spielräume für Ausnahmefälle zu, über die die Mehrheit der Mitglieder der beiden Kammern des Parlaments zusätzlich beschließen muss. Mit der Ausgabenregel wird die Höhe der zulässigen Ausgaben berechnet. Mit der Ausgleichsregel wird der Ausgleich zu hoher Ausgaben durch Kürzungen verpflichtend umgesetzt. Defizite, die sechs Prozent der im vorausgegangenen Rechnungsjahr getätigten Gesamtausgaben überschreiten, sind innerhalb der nächsten drei Jahre auszugleichen. Mit der Tilgungsregel wird der Staat verpflichtet, außerordentliche Einnahmen zur Tilgung der bereits bestehenden Schulden einzusetzen. Seit 2010 gilt eine Ergänzungsregel, die auch die Verschuldung außerhalb des ordentlichen Haushalts (also der „Schattenhaushalte") eindämmen soll. Erstmals 2010 führte die Schuldenbremse zu Budgeteinschnitten auf Bundesebene. ${ }^{8}$

Für das Umsetzen von Schuldenbremsen gibt es offensichtlich im Kontext des Föderalismus nicht nur unterschiedliche Lösungen, sondern auch unterschiedliche politische Präferenzen.

5 Vgl. OECD, Economic Surveys: United States, Paris 2010, S. 82 ff.: „A key factor contributing to the weakening of the fiscal position was the abandonment in 2002 of pay-as-you-go (PAYGO) budgeting strictures requiring deficit-neutrality for any new tax or spending initiative."

6 Lars P. Feld, Zu Ratio und Empirie von Schuldenschranken im föderalen Kontext, in: Ralf Thomas Baus / Annegret Eppler / Ole Wintermann (Hrsg.), a.a.O. (Fn. 1), S. 98 - 116, S. 108.

7 Vgl. Charles B. Blankart / Erik R. Fasten, Ein Optionsmodell für Schuldengrenzen im Föderalismus, in: Ralf Thomas Baus / Annegret Eppler / Ole Wintermann (Hrsg.), a.a.O. (Fn. 1), S. 147 153.

8 Vgl. Lars P. Feld, a.a.O. (Fn. 6), S. 108; Marco Duller, Föderalismusreform II: Lernen von der Schweiz, Marburg 2008; Jürgen Dunsch, Schweizer Schuldenbremse im Härtetest, in: FAZ vom 30. März 2010, S. 10. 


\section{Wie funktionieren Schuldenbremsen im Föderalismus?}

Vorausgeschickt sei die Bemerkung, dass der Rückgriff auf den Mechanismus der Schuldenbremse zuallererst ein Indiz für Politikversagen ist. Niemand hindert die politisch Verantwortlichen daran, entweder durch Steuererhöhungen oder Ausgabenkürzungen (oder beides) den Jahreshaushalt ausgeglichen zu gestalten. Fehlt hierzu der politische Wille oder die politische Kraft soll die Schuldenbremse als quasi neutrale Instanz (wie beispielsweise auch das Bundesverfassungsgericht in anderen Fragen) der Politik Grenzen setzen. Diese (künstliche) Externalisierung politischer Verantwortung hat bestenfalls wahltaktische Vorteile, weil dadurch der Regierung die direkte Zuschreibung unpopulärer Politik erspart bleiben soll, der Preis dieses Manövers ist aber hoch. Er besteht im Transparenz- und Responsivitätsverlust des politischen Entscheidungsprozesses, weil dieser auf dem Bürger unbekannte und für ihn undurchsichtige Mechanismen setzt, deren übergeordneter beziehungsweise unpolitischer Status ihm nicht einleuchtet.

Wenn eine Schuldenbremse eingerichtet wird, muss ihre Wirksamkeit und ihre Fähigkeit zur Defizitbegrenzung überzeugen. Also anders als der vorherige Artikel $115 \mathrm{GG}$, der Ausgaben zur Abwehr einer Störung des gesamtwirtschaftlichen Gleichgewichts als quasi Generalermächtigung zum Geldausgeben erlaubte und Ausgabengrenzen an einen weitgehend unbestimmten Investitionsbegriff band, was zudem sanktionslos ignoriert werden konnte, braucht eine Schuldenbremse „Zähne“. Sie braucht auch die Möglichkeit, für haushaltspolitische Entscheidungen, nicht nur auf der Ausgaben-, sondern auch auf der Einnahmenseite Verantwortung zu übernehmen, um wirksam Haushaltslücken füllen zu können. Deshalb kann die Schuldenbremse am besten in dualen föderalen Systemen mit haushaltspolitischer Eigenverantwortung der Gliedstaaten funktionieren und am schlechtesten in Systemen der Politikverflechtung mit mangelnder Einnahmen- und Ausgabenhoheit der Länder.

Es muss auch klar sein, dass der „no bail-out“-Grundsatz gilt, also unter normalen Umständen weder der Bund noch die Gemeinschaft der Gliedstaaten einem verantwortungslosen Land aus der Klemme hilft. Die „bündische Solidarität“ hat hier Grenzen. Für den Bund bedeutet eine Schuldenbremse auf allen politischen Ebenen zudem, dass seine politischen Initiativen nicht dazu führen dürfen, die Länder oder Kommunen in eine Kosten- oder Steuerfalle zu führen. Autonomie der Länder, Eigenverantwortung, eigene Gestaltungsmöglichkeiten von Einnahmen und Ausgaben, das klingt eher nach Wettbewerbsföderalismus als nach deutscher Politikverflechtung. Und nur diese Voraussetzungen würden es auch erlauben, zur politischen Disziplinierung der Haushaltspolitik der Länder noch die Disziplinierung durch die Märkte beziehungsweise durch die Rating-Agenturen hinzuzufügen. Landesregierungen, die ihren Haushalt nicht ausgleichen, müssten dann im Idealfalle nicht nur ihren Bürgern gegenüber höhere Steuern oder niedrigere Ausgaben verantworten, wenn sie Haushalte mit Finanzierungslücken beschließen, sondern auch auf den Märkten wegen schlechterer Kreditwürdigkeit für ihre angehäuften Schulden höhere Zinsen zahlen.

Wenn im Föderalismus eine Schuldenbremse für die Länder eingeführt wird, wäre dieses Vorhaben zuerst in den Landesparlamenten zu diskutieren. Freiwilligkeit und bottom upEntscheidungsprozesse schaffen erst das nötige Problembewusstsein und die unabdingbare Legitimation bei Politik und Bürgern für eine solch einschneidende Restriktion des Bedarfsprinzips bei der Budgetierung. Die Landesparlamente haben das Haushaltsrecht ge- 
mäß den Landesverfassungen. Sinnvoll wäre eine Änderung in den Landesverfassungen, die einen ohne Defizitausnahme ausgeglichenen Haushalt zwingend vorschreibt. In Hessen und Bayern sind für eine solche Verfassungsänderung Volksabstimmungen erforderlich. Aber auch wenn dies die Verfassung nicht vorschreibt, wäre dem Landesvolk dieser folgenreiche Schritt für das Gemeinwesen entsprechend intensiv politisch zu vermitteln.

In Deutschland fühlten sich die Landtage vor der Verabschiedung der Föderalismusreform II nicht angesprochen. Was in Kanada oder den USA für die Gliedstaaten selbstverständlich ist und weitgehend durch eigenständige „balanced budget“-Klauseln in ihren Verfassungen umgesetzt wurde, wird den deutschen Landesparlamenten erst jetzt langsam klar. ${ }^{9}$ Die Chance einer bottom-up Lösung wurde weitgehend verspielt, ja noch nicht einmal ernsthaft erwogen. In Deutschland akzeptierte man, dass sich die Landesparlamente in der Verschuldungsfrage ihr historisch vornehmstes Recht, das Budgetrecht, von den Landesregierungen nehmen ließen, die wiederum im Bundesrat einer Grundgesetzänderung zustimmten und keine Probleme mit einer bundesgesetzlichen Regelung des ausgeglichenen Haushalts der Länder ohne Einbindung der Landtage hatten. Nur Schleswig-Holstein hat sich verweigert und 2010 eine Klage beim Bundesverfassungsgericht eingereicht. Es macht geltend, dass das Vorgehen bei der Föderalismusreform II gegen Artikel 109, Absatz 1 GG verstößt, der die Unabhängigkeit und Selbständigkeit der Länder in ihrer Haushaltswirtschaft garantiert. Damit werde auch das Bundesstaatsprinzip (Artikel 20, Absatz 1 GG) verletzt. Über die Klage ist noch nicht entschieden.

Inzwischen ist die Diskussion über die Folgen der Schuldenbremse auch auf der Länderebene angekommen. Drei unterschiedliche politische und verfahrenstechnische Strategien lassen sich unterscheiden: (1) Die Aufnahme der Verpflichtung des Landes, den Haushalt ohne Kreditaufnahme auszugleichen, in die Landesverfassung (vier Länder); (2) die Aufnahme dieser Festlegung in die Landeshaushaltsordnung (sechs Länder) und (3) das Ignorieren der Notwendigkeit einer landesgesetzlichen Regelung aus unterschiedlichen Motiven (sechs Länder).

Parteipolitisch sind diese drei Strategien nicht zuzuordnen (vgl. Tabelle 1). Sie hängen sehr stark von landespolitischen Interessen, bevorstehenden Wahlen und parlamentarischen Mehrheiten ab. Zwar sind CDU und FDP meist für die Anpassung der jeweiligen Landesverfassung an die Vorgaben des Grundgesetzes, und die SPD wendet sich dagegen - aber dies ist in wenigen Ländern auch umgekehrt der Fall. Nur „Die Linke“ und der DGB wenden sich in jedem Land gegen eine Festlegung von Ausgabengrenzen für den Staat. Sie sehen darin eine unnötige Einschränkung der wirtschaftspolitischen Handlungsmöglichkeiten des Staates und erwarten als Folge einer Sparpolitik die Kürzung von Sozialleistungen, was sie ablehnen. Wirtschaftspolitisch wird die Gegnerschaft zur Schuldenbremse mit dem Hinweis auf keynesianische Steuerungsstrategien begründet.

Vier Länder haben sich für eine Aufnahme der Schuldenbremse in ihre Landesverfassung entschieden. Schleswig-Holstein folgt bei der Ausgestaltung der Logik der Vorgaben für Konsolidierungsländer, also dem stufenweisen Abbau der Nettoneuverschuldung um jährlich zehn Prozent. Das Land lässt Ausnahmen beim jährlichen Schuldenabbau, die der Konsolidierungspflicht ja widersprechen würden, nur bei einer Zweidrittelmehrheit im

9 Vgl. Roland Sturm, Regeln die Länder ihre Haushaltspolitik neu? Reaktionen auf den Zwang zum Haushaltsausgleich durch die Föderalismusreform II, in: Gesellschaft - Wirtschaft - Politik, 60. Jg. (2011), H. 2, S. $165-170$. 


\begin{tabular}{|c|c|c|c|c|}
\hline \multicolumn{5}{|c|}{ Tabelle 1: Die Schuldenbremse als Gegenstand von Parteipolitik } \\
\hline Land & Dafür & Dagegen & $\begin{array}{c}\text { Gesetzliche } \\
\text { Neuregelung }\end{array}$ & \begin{tabular}{|l} 
Verschuldung 2009 \\
(in Millionen Euro)
\end{tabular} \\
\hline Baden-Württemberg & SPD & CDU, FDP & Keine & 56.415 \\
\hline Bayern & FDP & Keine Debatte & Keine & 27.567 \\
\hline Berlin & Keine & Debatte & Keine & 58.821 \\
\hline Brandenburg & $\begin{array}{l}\text { CDU, FDP, } \\
\text { Grüne }\end{array}$ & \begin{tabular}{|l|} 
SPD, \\
Die Linke \\
\end{tabular} & Keine & 17.433 \\
\hline Bremen & $\begin{array}{l}\text { SPD, Grüne, } \\
\text { CDU }\end{array}$ & Die Linke & $\begin{array}{l}\text { Geplante Änderung } \\
\text { der LV }\end{array}$ & 16.011 \\
\hline Hamburg & GAL, SPD & $\mathrm{CDU}$ & Keine & 23.879 \\
\hline Hessen & $\begin{array}{l}\text { CDU, FDP, } \\
\text { SPD, Grüne }\end{array}$ & Die Linke & $\begin{array}{l}\text { LV Art. } 141 \text { in Ver- } \\
\text { bindung mit Art. } 161\end{array}$ & 33.406 \\
\hline $\begin{array}{l}\text { Mecklenburg- } \\
\text { Vorpommern }\end{array}$ & $\begin{array}{l}\text { CDU, SPD, } \\
\text { FDP }\end{array}$ & Die Linke & $\begin{array}{l}\text { LV Art. 65(2) in Ver- } \\
\text { bindung mit Art. } 79 a\end{array}$ & 9.905 \\
\hline Niedersachen & CDU, FDP & \begin{tabular}{|l|} 
SPD, \\
Die Linke \\
\end{tabular} & Keine & 51.461 \\
\hline Nordrhein-Westfalen & CDU, FDP & \begin{tabular}{|l|} 
SPD, Grüne, \\
Die Linke \\
\end{tabular} & Keine & 122.061 \\
\hline Rheinland-Pfalz & $\begin{array}{l}\text { CDU, SPD, } \\
\text { FDP }\end{array}$ & Keine Partei & LV Art.117 (ab 2012) & 28.455 \\
\hline Saarland & Keine & Debatte & Keine & 10.593 \\
\hline Sachsen & CDU, FDP & $\begin{array}{l}\text { SPD, } \\
\text { Die Linke }\end{array}$ & $\begin{array}{l}2009 \text { Übernahme der } \\
\text { GG-Bestimmung Art. } \\
109(3) \text { in } \$ 18 \text { LHO }\end{array}$ & 6.889 \\
\hline Sachsen-Anhalt & CDU, SPD & Die Linke & $\begin{array}{l}2009 \text { Übernahme der } \\
\text { GG-Bestimmung Art. } \\
109(3) \text { in } \$ 18 \text { LHO }\end{array}$ & 19.812 \\
\hline Schleswig-Holstein & $\begin{array}{l}\text { CDU, FDP, } \\
\text { SPD, Grüne, } \\
\text { SSW }\end{array}$ & Die Linke & LV Art. 49 & 25.422 \\
\hline Thüringen & CDU, FDP & $\begin{array}{l}\text { Die Linke, } \\
\text { SPD }\end{array}$ & $\begin{array}{l}2009 \text { Übernahme der } \\
\text { GG-Bestimmung Art. } \\
109(3) \text { in } \$ 18 \text { LHO }\end{array}$ & 15.707 \\
\hline \multicolumn{5}{|c|}{$\begin{array}{l}\text { Anmerkung: Kursiv gesetzt sind Länder mit Schuldenbremse in ihrer Verfassung (beziehungsweise ge- } \\
\text { plant). } \\
\text { Quelle: Eigene Recherche und Statistisches Bundesamt: Statistisches Jahrbuch 2010, Wiesbaden 2010, } \\
\text { S. } 568 .\end{array}$} \\
\hline
\end{tabular}

Landtag zu (Artikel 49). In Hessen fand am 27. März 2011 mit den dortigen Kommunalwahlen ein Volksentscheid statt, der für die Veränderung der Landesverfassung (Artikel 141) erforderlich war. Bei einer Wahlbeteiligung von 48,9 Prozent sprachen sich 70 Prozent der Abstimmenden für die Aufnahme der Schuldenbremse in die Hessische Verfassung aus. Die Schuldenbremse bindet den Gesetzgeber dort aber erst ab 2020 (Artikel 161). Begründet wird dies „mit den massiven Verwerfungen, die die Wirtschafts- und Finanzkrise im Landeshaushalt hinterlassen hat ${ }^{\text {"10 }} .2020$ greift aber ohnehin die Grundgesetzbestimmung.

10 Hessisches Ministerium der Finanzen, Die Einführung der Schuldenbremse, Hintergründe und Argumente, Wiesbaden 2010, S. 17. 
SPD und Grüne haben für ihre Unterstützung der Verfassungsänderung einen Zusatz zur Schuldenbremse verlangt; danach soll sie „ungeachtet der Einnahmen- und Ausgabenverantwortung des Landtages und der Landesregierung" gelten. Die Interpretation dieses $\mathrm{Zu}$ satzes ist umstritten. Entweder ist er überflüssig, weil er Selbstverständliches festschreibt, oder die Einnahmen- und Ausgabenverantwortung des Landtages und der Landesregierung soll betont werden, was den Verdacht nahe legt, die Anwendung der Schuldenbremse solle eingeschränkt werden. Argumentiert wird auch, dass die Verfassungsänderung das Landesparlament entmachtet, weil sie „Einnahmen aus Krediten“ verbietet, nicht aber die „Aufnahme von Krediten". Hier könne die Landesregierung alternative Finanzierungsformen nutzen, ohne das Parlament zu fragen. ${ }^{11}$

In der Rheinland-Pfälzischen Landesverfassung (Artikel 117) findet sich eine Schuldenbremse, die ab 2012 verbindlich ist. Dies ist - anders als in Hessen - eine unmittelbare Anerkennung des neuen Sparzwangs. Allerdings befürchteten SPD und FDP, dass der Bund einseitig Einnahmeausfälle des Landes verursachen könnte, weshalb die Schuldenbremse die Ausnahmebestimmung enthält, dass in einem solchen Falle auf vier Jahre befristet vom ausgeglichenen Haushalt abgewichen werden könne. Die CDU lehnte diese Einschränkung ab. Mecklenburg-Vorpommern hat 2011 seine Landesverfassung geändert. 50 von 71 Abgeordneten stimmten für eine Änderung des Artikels 65 Absatz 2. Der Wortlaut des neuen Artikels orientiert sich am Artikel 109 Absatz 3 des Grundgesetzes. Die Festlegung auf einen ausgeglichenen Haushalt gilt ab 2020. Ein neuer Artikel 79A in der Landesverfassung bestimmt, dass die Haushalte bis zum Haushaltsjahr 2019 so aufzustellen sind, dass die Vorgabe des neuen Artikels 65(2) ab 2020 erfüllt wird.

Sechs Länder verweisen bei der Umsetzung der Verpflichtung zu einem ausgeglichenen Haushalt auf ihre Landeshaushaltsordnungen. Nur drei von ihnen, sämtlich ostdeutsche Länder (Sachsen, Sachsen-Anhalt und Thüringen), nehmen aber die Vorgaben des Grundgesetzes auf. Seit 2009 findet sich in den Landeshaushaltsordnungen die Übernahme der GG-Bestimmung in Artikel 109, Absatz 3: „Der Haushaltsplan ist ohne Einnahmen aus Krediten auszugleichen" mit den zulässigen Ausnahmen. ${ }^{12}$ Thüringen hat, wie RheinlandPfalz, eine weitere Ausnahmeklausel erfunden, die sich auf vom Bund verursachte Einnahmeausfälle bezieht, wobei hier der Durchschnitt der letzten drei Haushaltsjahre zugrunde gelegt wird.

Bayern, Baden-Württemberg und Hamburg, die zu den finanzstarken Ländern zählen, sind in ihrer politischen Praxis dem ausgeglichenen Haushalt nicht abgeneigt. Bayern beansprucht hier sogar eine Vorreiterrolle. Eine Notwendigkeit, ihre Haushaltsordnungen zu ändern, sehen diese Länder aber nicht, obwohl diese weiterhin die inzwischen aus dem Grundgesetz (Artikel 115 alt) gestrichene Generalermächtigung enthalten, bei einer „Störung des gesamtwirtschaftlichen Gleichgewichts" die Schuldenbremse ignorieren zu dürfen.

Die Stellungnahme des bis Mai 2011 CDU-geführten baden-württembergischen Finanzministeriums zur Anfrage der SPD im Landtag, ob und in welcher Form die Schuldenbremse in die Landesverfassung aufgenommen werden soll, spricht für sich: „Der Landes-

11 Vgl. Henning Tappe, Das Parlament wird entmündigt, in: FAZ vom 24. März 2011, S. 8.

12 Ausnahmen: Erstens eine von der Normallage abweichende konjunkturelle Entwicklung, die die Finanzlage des Landes nicht unerheblich beeinträchtigt und zweitens Naturkatastrophen oder außergewöhnliche Notsituationen, die sich der Kontrolle des Staates entziehen und die Finanzlage des Landes erheblich beeinträchtigen. 
gesetzgeber hat mit $\$ 18$ LHO bereits eine Regelung geschaffen, die zwar mit Blick auf die ab 2020 vorbehaltlos geltende Vorgabe des Grundgesetzes anpassungsbedürftig ist, die aber nach Sinn und Zweck der durch die grundgesetzliche Vorgabe intendierten Schuldenbegrenzung im Grundsatz entspricht. Aus Sicht der Landesregierung ist deshalb die Anpassung der landesrechtlichen Vorgaben - auch angesichts des grundgesetzlich vorgesehenen Übergangszeitraums - nicht vordringlich. Eine Neuregelung sollte vielmehr erst auf der Grundlage der Erfahrungen mit der gegenwärtigen Finanz- und Wirtschaftskrise und deren Überwindung aufbauen. Solange die Auswirkungen der Finanz- und Wirtschaftskrise fortdauern, ist hierfür noch nicht der richtige Zeitpunkt [gekommen]. Dies gilt für BadenWürttemberg umso mehr, da wegen der exportorientierten Wirtschaftsstruktur konjunkturelle Ausschläge nach oben wie unten in der Regel stärker ausfallen als in anderen Ländern. So bedarf zum Beispiel die Ausgestaltung einer Konjunkturkomponente besonderer Prüfung. Vor einem Vorschlag für eine Änderung der Landesverfassung sollte daher die weitere wirtschaftliche Entwicklung abgewartet werden, damit eine zukunftsorientierte und nachbesserungsfeste Regelung geschaffen wird. "13 Die neue grün-rote Landesregierung plant einen schuldenfreien Haushalt frühestens 2019.

Kein landespolitisches Thema ist die Schuldenbremse in den zwei Konsolidierungsländern Berlin und Saarland. Hier verlässt man sich auf die Vorgaben des Bundes. Die Grundhaltung in diesen Ländern, die schon bisher immer vom Bund erwarteten, ihnen in haushaltspolitisch schwierigen Zeiten zu helfen, hat sich damit nicht geändert. Bremen und das Saarland haben in der Vergangenheit erfolgreich eine „haushaltspolitische Notlage“ geltend gemacht und zu deren Beseitigung spezielle Bundesergänzungszuweisungen im Finanzausgleich erhalten. Berlin scheiterte mit einem ähnlichen Ansinnen beim Bundesverfassungsgericht. Für Berlin und das Saarland ist der ausgeglichene Haushalt, anders als in den Konsolidierungsländern Sachsen-Anhalt und Schleswig-Holstein und nunmehr auch Bremen, weniger eine landespolitische Angelegenheit als ein Konfliktfeld mit dem Bund. In ihrem Fokus stehen die vertikalen Finanzbeziehungen und mögliche Verhandlungslösungen in der Auseinandersetzung mit der Bundesregierung. Die 2011 ins Amt gekommene rot-grüne Bremer Landesregierung hat eine Initiative zur Verankerung der Schuldenbremse in der Landesverfassung angekündigt.

In Brandenburg und Niedersachsen fehlen die notwendigen parlamentarischen Mehrheiten für eine Änderung der Landesverfassung, weshalb hier die Debatte zum ausgeglichenen Haushalt mehr oder minder „eingeschlafen“ ist. Im März 2011 billigten in Niedersachsen CDU und FDP im Kabinett den Entwurf von zwei neuen Artikeln der Landesverfassung, die die Schuldenbremse ab 2017 einführen würden und wie in Schleswig-Holstein für Abweichungen von dieser Bestimmung die Hürde von Zweidrittelmehrheiten vorsehen. In Brandenburg verhindern die Regierungsparteien SPD und Die Linke schon eine Änderung der Landeshaushaltsordnung. In Niedersachsen blockieren dieselben Parteien die erforderliche verfassungsändernde Mehrheit.

Ein Sonderfall beim Umgang mit den Anforderungen der Schuldenbremse an die Landespolitik stellt Nordrhein-Westfalen dar. Auch hier gibt es keine parlamentarische Mehr-

13 Landtag von Baden-Württemberg, Antrag der Fraktion der SPD und Stellungnahme des Finanzministeriums, Umsetzung der Schuldenbremse nach den Regelungen in der Landeshaushaltsordnung, in: Drs. 14/6223 vom 19. April 2010, http://www.landtag-bw.de/WP14/Drucksachen/ 6000/14_6223_d.pdf (Abruf am 30. Mai 2011). 
heit für die Schuldenbremse, so dass bisher zwei Anläufe von CDU und FDP in der Regierung und in der Opposition scheiterten, die Landesverfassung entsprechend zu ändern. Die derzeitige Ministerpräsidentin Hannelore Kraft (SPD) geht sogar davon aus, dass auch der Bund 2016 die Schuldenbremse nicht wie geplant einhalten wird. ${ }^{14}$ Die Lage hat sich noch dadurch verschärft, dass die rot-grüne Landesregierung 2010 einen - auch nach den bisherigen Verschuldungsgrenzen - verfassungswidrigen Haushalt vorgelegt hat. Die Summe der Kredite überstieg die Summe der Investitionen. Die nachgeschobene Begründung, es gehe darum, eine Störung des gesamtwirtschaftlichen Gleichgewichts zu beheben, war für die damalige wirtschaftliche Aufschwungperiode wenig glaubhaft. Das Landesverfassungsgericht bestätigte deshalb die Verfassungswidrigkeit des Landeshaushalts. Auch gegen den Landeshaushalt 2011 hat die CDU Klage beim Verfassungsgericht in Münster eingelegt. Die Frage bleibt offen, wie die Landesregierung in Nordrhein-Westfalen den Defizitabbau im Hinblick auf 2020 gestalten will.

\section{Wie funktioniert die Schuldenbremse in Deutschland?}

Das Ende der Neuverschuldungspolitik soll für den Bund 2016 kommen, für die Länder 2020. Die Mechanik der Schuldenbremse, so wie sie ins Grundgesetz geschrieben wurde, ist aus mehreren Gründen bemerkenswert: zum einen, wie erwähnt, wegen der verfassungsrechtlich zweifelhaften Idee, den Landeshaushalten und damit dem Landesgesetzgeber durch eine bundesgesetzliche Regelung Grenzen zu setzen; zum zweiten überrascht die Wiederbelebung des Keynesianismus samt dem Anspruch, Konjunkturzyklen beherrschen zu können - eine Idee, die schon einmal aufgegeben wurde, als die Tatsache, dass nationale Volkswirtschaften angesichts der Globalisierung eine Fiktion sind, noch weit weniger Allgemeingut war und der Europäische Binnenmarkt sowie der Euro noch in weiter Ferne lagen. Der neue Glaube an die Volkswirtschaftslehre, die ihre Präzision und ihre Prognosekraft in der Finanzkrise nicht gerade überzeugend bewiesen hat, schimmert auch durch viele Details der Regelung, wie im einzelnen noch darzulegen sein wird.

Bei den Wirkungen der Schuldenbremse ist zu unterscheiden zwischen der Übergangsperiode (für den Bund bis 2016 und für die Länder bis 2020) und der Zeit danach. In der Übergangsphase gilt für die Länder, dass sie im Zeitraum vom 1. Januar 2011 bis zum 31. Dezember 2019 frei entscheiden, welchen konkreten Pfad sie zum Abbau ihrer jährlichen Finanzierungsdefizite wählen. Allerdings müssen alle im Haushaltsjahr 2020 einen ausgeglichen Haushalt ohne Kreditfinanzierung vorlegen. Für Bremen (300 Millionen Euro jährlich), das Saarland (260 Millionen), Berlin, Sachsen-Anhalt und Schleswig-Holstein (je 80 Millionen) sind so genannte Konsolidierungshilfen vorgesehen, die sich für 2011 bis 2019 jährlich auf insgesamt 800 Millionen Euro belaufen. Sie werden je zur Hälfte vom Bund und den Ländern (aus ihrem Umsatzsteueranteil) getragen. Voraussetzung für die Gewährung der Konsolidierungshilfe und damit der Solidarität der Geberländer und des Bundes ist, dass die Empfängerländer tatsächlich bis 2020 ihre jährliche Neuverschuldung auf Null bringen. Hinzu kommt, dass das Grundgesetz die gleichzeitige Gewährung der Konsolidierungshilfen und von Sanierungshilfen im Wege der Bundesergänzungszuweisungen an 


\begin{tabular}{|c|c|c|}
\hline & Bund & Länder \\
\hline Nullverschuldung & $\begin{array}{l}\text { bei einer Verschuldung von } 0,35 \\
\text { Prozent des BIP bereits erreicht }\end{array}$ & $\begin{array}{l}\text { Nullverschuldung heißt } \\
\text { Nullverschuldung }\end{array}$ \\
\hline Nullverschuldung ab & 2016 & 2020 \\
\hline Ausnahmen & $\begin{array}{l}\text { bei Nachtragshaushalten wegen } \\
\text { Abweichungen im Vollzug des } \\
\text { Haushalts weiterer Spielraum } \\
\text { von } 3 \text { Prozent der veranschlagten } \\
\text { Steuereinnahmen }\end{array}$ & keine \\
\hline Konjunkturkomponente & $\begin{array}{l}\text { erlaubt Verschuldung } \\
\text { von } 1,5 \text { Prozent des BIP }\end{array}$ & $\begin{array}{l}\text { ja, als temporäre Abweichung } \\
\text { von Nullverschuldung }\end{array}$ \\
\hline Übergangsregeln & keine & $\begin{array}{l}\text { Konsolidierungshilfen für Bremen, } \\
\text { Saarland, Berlin, Sachsen-Anhalt } \\
\text { und Schleswig-Holstein; jährlich } \\
800 \text { Millionen Euro; finanziert } \\
\text { aus Umsatzsteueranteilen } \\
\text { von Bund und Ländern } \\
\end{array}$ \\
\hline Sanktionen vor 2020 & noch zu regeln & $\begin{array}{l}\text { noch zu regeln; für die Empfänger } \\
\text { der Konsolidierungshilfen: Verwar- } \\
\text { nung; Zurückhalten von einem } \\
\text { Drittel der jährlichen Zahlung } \\
\text { beziehungsweise Rückzahlung der } \\
\text { erhaltenen zwei Drittel }\end{array}$ \\
\hline $\begin{array}{l}\text { Sanktionen nach } 2016 \\
\text { beziehungsweise } 2020\end{array}$ & $\begin{array}{l}\text { Sanierungsverfahren über fünf Jahre } \\
\text { durch Vereinbarung mit dem Stabili- } \\
\text { tätsrat; halbjährliche Berichtspflicht; } \\
\text { bei Fehlverhalten Aufforderung zur } \\
\text { Besserung; nach einem Jahr erneute } \\
\text { Aufforderung; neues Sanierungspro- } \\
\text { gramm etc. }\end{array}$ & $\begin{array}{l}\text { Sanierungsverfahren über fünf Jahre } \\
\text { durch Vereinbarung mit dem Stabili- } \\
\text { tätsrat; halbjährliche Berichtspllicht; } \\
\text { bei Fehlverhalten Aufforderung zur } \\
\text { Besserung; nach einem Jahr erneute } \\
\text { Aufforderung; neues Sanierungspro- } \\
\text { gramm etc. }\end{array}$ \\
\hline \multicolumn{3}{|c|}{$\begin{array}{l}\text { Anmerkung: Zu Details vgl. die Beschlüsse der Kommission von Bundestag und Bundesrat zur Moder- } \\
\text { nisierung der Bund-Länder-Finanzbeziehungen vom 5. März 2009, Kommissionsdrucksache } 174 . \\
\text { Quelle: Roland Sturm, Föderalismusreform II: „Schuldenbremse“, neokeynesianischer Glaube an die } \\
\text { Steuerbarkeit der Wirtschaft und das altbekannte Instrument der Politikverflechtung, in: Gesellschaft - } \\
\text { Wirtschaft - Politik, 58. Jg. (2009), H. 4, S. } 487 \text { - 499, S. } 492 \text {. }\end{array}$} \\
\hline
\end{tabular}

Länder in einer extremen Haushaltsnotlage ausschließt. Dies war ein Wink mit dem Zaunpfahl an die Adresse Bremens und des Saarlands, die damit implizit aufgefordert wurden, ihre Klage vor dem Bundesverfassungsgericht auf Anerkennung einer extremen Haushaltsnotlage für erledigt zu erklären.

Überwacht wird das Wohlverhalten der Länder, die Konsolidierungshilfen erhalten, durch den Stabilitätsrat (zu dieser neuen Einrichtung später mehr). Seiner Tätigkeit liegt ein einfacher Rechenmechanismus zugrunde: Es werden jährliche Obergrenzen für den Defizitabbau festgelegt. 2011 bis 2020 sind zehn Haushaltsjahre. Die Obergrenze für 2011 errechnet sich, indem das strukturelle Finanzierungsdefizit des Jahres 2010 um ein Zehntel verringert wird. Für die Folgejahre wird die Obergrenze des Vorjahres jeweils um ein Zehntel verringert. Allerdings kann der Stabilitätsrat, ohne das letztendliche Ziel der Nullverschuldung 2020 aufzugeben, in begründeten Fällen eine Überschreitung der Obergrenzen 
erlauben. Die Auszahlung der Jahresbeträge der Konsolidierungshilfen erfolgt durch das Bundesfinanzministerium in Höhe von zwei Dritteln zum 1. Juli des laufenden Jahres. Der Stabilitätsrat entscheidet, ob die Voraussetzungen für die Auszahlung der Gesamtsumme gegeben sind. Das restliche Drittel wird zum 1. Juli des Folgejahres ausgezahlt. Werden die Voraussetzungen nicht erfüllt, sind auch die bereits ausbezahlten zwei Drittel zurückzuzahlen. Verfehlt ein Land sein Konsolidierungsziel in einem Jahr, verliert es den Anspruch auf Konsolidierungshilfe für dieses Jahr und wird vom Stabilitätsrat verwarnt. Falls dieses Land in einem späteren Jahr die Regeln wieder einhält, erhält es entsprechende Zahlungen. Hilfszahlungen für vergangene Jahre, auf die kein Anspruch bestand, werden nicht nachgeholt.

Für den Bund gilt in der Übergangsphase, dass er frei in deren Gestaltung ist, aber bis 2016 das Ziel einer konjunkturell bereinigten jährlichen Nettoneuverschuldung von höchstens 0,35 Prozent des BIP erreichen muss. Die Übergangsperiode läuft also nach drei Regelsystemen ab: Bund, Länder ohne Konsolidierungshilfen und Länder mit Konsolidierungshilfen.

Nach 2020 gilt ein Verschuldungsverbot der Länder. Der Bund darf sich jährlich um höchstens 0,35 Prozent des BIP verschulden. Überwacht wird die haushaltspolitische Solidität durch den 2010 neu geschaffenen bei der Bundesregierung angesiedelten Stabilitätsrat. ${ }^{15}$ Diesem gehören an: der Bundesminister der Finanzen, die für die Finanzen zuständigen Minister der Länder und der Bundesminister für Wirtschaft und Technologie. Den Vorsitz führen gemeinsam der Bundesminister der Finanzen und der Vorsitzende der Länderfinanzministerkonferenz. Der Stabilitätsrat tritt mindestens zweimal jährlich zusammen. Seine Beschlüsse werden mit der Stimme des Bundes und der Mehrheit von zwei Dritteln der Länder gefasst. Bei Entscheidungen, die ein bestimmtes Land betreffen, ist dieses nicht stimmberechtigt. Bei Entscheidungen, die den Bund betreffen, genügt die Mehrheit von zwei Dritteln aller stimmberechtigten Mitglieder.

Der Stabilitätsrat beschließt allgemein geltende Schwellenwerte für die einzelnen von ihm festgelegten Kennziffern, deren Überschreitung auf eine drohende Haushaltsnotlage hinweisen. Stellt der Stabilitätsrat eine drohende Haushaltsnotlage fest, vereinbart er mit dem Bund oder Land ein Sanierungsprogramm, das sich grundsätzlich über einen Zeitraum von fünf Jahren erstreckt (kürzere Zeiträume sind möglich). Es enthält Vorgaben über die angestrebten Abbauschritte der jährlichen Nettokreditaufnahme und die geeigneten Sanierungsmaßnahmen. Geeignet heißt in diesem Zusammenhang, dass sie allein aus der betroffenen Gebietskörperschaft kommen. Diese berichtet dem Stabilitätsrat halbjährlich. Misslingt die Sanierung, sucht der Stabilitätsrat im Einvernehmen mit der betroffenen Gebietskörperschaft nach neuen Wegen. Legt der Bund oder das Land ungeeignete oder unzureichende Vorschläge für Sanierungsmaßnahmen vor oder setzt die Gebietskörperschaft die vereinbarten Maßnahmen nur unzureichend um, beschließt der Stabilitätsrat eine Aufforderung zur verstärkten Haushaltssanierung. Spätestens ein Jahr nach dieser Aufforderung prüft der Stabilitätsrat, ob die nötigen Maßnahmen zur Haushaltssanierung ergriffen wurden. Ist dies nicht der Fall, ergeht eine neue Aufforderung. Erweist sich das Sanierungsprogramm als unzureichend, wird ein neues aufgelegt. Der Stabilitätsrat hat also bei Nichtbeachten der Budgetdisziplin lediglich das Mittel der Aufforderung, analog zum „Blauen Brief“, den die EU-Kommission jenen Mitgliedstaaten zukommen lässt, die das

15 Stabilitätsratsgesetz-StabiRatG vom 25. Juni 2010. Die konstituierende Sitzung des Stabilitätsrats fand am 28. April 2010 statt. 
Drei-Prozent-Maastricht-Kriterium nicht einhalten. Anders als die EU-Kommission kann der Stabilitätsrat aber keine Geldstrafen verhängen.

Das Grundgesetz unterscheidet zwischen der strukturellen und der konjunkturellen Verschuldung, allerdings in einer Sprache, die dem Gedanken der Lesbarkeit einer Verfassung für alle Bürger deutlich widerspricht. Im neuen Art. 115 Absatz 2 GG findet sich der Satz: „Zusätzlich sind bei einer von der Normallage abweichenden konjunkturellen Entwicklung die Auswirkungen auf den Haushalt im Auf- und Abschwung symmetrisch zu berücksichtigen." In verständlicherer Sprache heißt das, der Bund soll ein prozyklisches, also den konjunkturellen Auf- oder Abschwung verstärkendes Ausgabenverhalten vermeiden. Dem wird dadurch Rechnung getragen, dass in konjunkturell problematischen Zeiten die jährliche Neuverschuldung des Bundes 1,5 Prozent betragen darf. Der notorischen Unfähigkeit der Politik, solche Mehrausgaben in konjunkturell besseren Zeiten zurückzuführen, wird durch Verbuchung der Abweichungen auf einem Kontrollkonto entgegengewirkt, das Rückzahlungspflichten begründet.

Ausnahmen von der Nullverschuldungsregel sind nur vorgesehen im Falle von Naturkatastrophen (hier lehnt sich die Regel an die Handhabung der Maastricht-Kriterien an) oder außergewöhnlichen Notsituationen, die sich der staatlichen Kontrolle entziehen und die staatliche Finanzlage erheblich beeinträchtigen (Beispiele: Finanzkrise, deutsche Einheit). Beschließt der Bundestag mit der Mehrheit seiner Mitglieder, dass es sich um eine Notsituation handelt, die ein Aussetzen der Nullverschuldungsregel erlaubt, muss er diesen Beschluss mit einem Tilgungsplan verbinden. Dieser Plan soll die Rückführung der aufgenommenen Kredite binnen eines angemessenen Zeitraums darlegen.

\section{Die deutsche Schuldenbremse: Effizient und föderalismusschonend?}

Ein endgültiges Urteil über die Effizienz der deutschen Schuldenbremse ist sicher verfrüht. Es lassen sich aber einige Erwartungen formulieren, die nicht zuletzt auf Erfahrungen mit den Prioritätensetzungen in der deutschen Politik und dem Wissen um die Logik institutionellen Handelns beruhen. Selbst wenn in dieser Perspektive die Schuldenbremse im Detail sich als ineffizient oder widersprüchlich erweist, ist nicht auszuschließen, dass allein ihre Existenz politische Diskurse und Entscheidungen weitgehend beeinflusst.

Im Hinblick auf Effizienzüberlegungen tauchen selbst bei wohlwollenden Beobachtern wie der OECD Bedenken auf. Da ist zunächst die Frage, ob der Stabilitätsrat richtig konstruiert ist angesichts des Grundgedankens der deutschen Schuldenbremse, die Entscheidung über die Defizitgrenze und deren Überwachung durch Einbeziehen einer neutralen Instanz, den Stabilitätsrat, zu externalisieren. Als „neutral“ lässt sich der Stabilitätsrat, so die OECD, sicher nicht bezeichnen: „In seiner derzeitigen Zusammensetzung gehören dem Rat jedoch die Finanzminister von Bund und Ländern an, so dass die Bundesregierung und die Landesregierungen sich im Wesentlichen selbst kontrollieren. Das könnte dazu führen, dass der Rat zu lax auf Haushaltsüberschreitungen oder Haushaltskosmetik reagiert. Um den Erfolg des Frühwarnsystems zu gewährleisten, sollte der Rat gestärkt werden, möglicherweise durch die Verwendung von Beiträgen unabhängiger Sachverständiger. "16 
Im Stabilitätsrat bieten sich zwei Diskurse zu Defizitgrenzen an, der ökonomische und der politische. Soll Beliebigkeit, Kompromiss und kleinster gemeinsamer Nenner einschließlich des Verbiegens der Haushaltsregeln vermieden werden, wäre der ökonomischen Argumentation Priorität zu geben, die auch beim Ruf der OECD nach unabhängigen Sachverständigen durchscheint. Ob es ,unabhängige Sachverständige“ gibt beziehungsweise geben kann, sei dahin gestellt. Größere Bedenken sollte man haben, dass diese Argumentation dazu führt, einseitig Volkswirten das letzte Wort bei Haushaltsentscheidungen zu geben. $\mathrm{Ob}$ diese tatsächlich über wissenschaftlich stichhaltige Erkenntnisse verfügen, darf angesichts ihrer langen Liste von Irrtümern bei der Politikberatung mit Fug und Recht bezweifelt werden. Den generellen Vorbehalt gegenüber der prognostischen Kraft der Wirtschaftswissenschaft hat Gideon Rachman kürzlich wieder einmal in der Financial Times formuliert: "The vanity of economists needs to be challenged. Above all, their claim to scientific rigour - buttressed by models and equations - must be treated much more sceptically." ${ }^{17}$

Im Kontext der Schuldenbremse heißt dies konkret: Diese baut nicht zuletzt auf einer keynesianischen Logik auf. Es sei Aufgabe des Staates, in Zeiten konjunktureller Abschwünge durch Ausgabenpolitik gegenzusteuern und in Zeiten wirtschaftlichen Booms Kaufkraft abzuschöpfen, um Inflation zu vermeiden und die Schulden aus der Krisenbekämpfung zurückzuzahlen. An letzterem, dem Schuldenabbau in wirtschaftlich guten Zeiten, ist die Politik in der Vergangenheit (zuletzt die zweite Große Koalition) regelmäßig gescheitert. Die keynesianische Logik verlangt dem Stabilitätsrat ab, den genauen Ort der Wirtschaftsentwicklung im Konjunkturverlauf zu bestimmen, auch wenn nationale Konjunkturentwicklungen im Zeitalter globalisierter Volkswirtschaften nur noch Fiktionen sind. Abweichungen von der Nullverschuldungsregel aus konjunkturellen Gründen sind nicht einfach später auszugleichen, sondern „konjunkturgerecht zurückzuführen“. Der Mythos des konjunkturell ,atmenden“ Haushalts setzt ganz auf wirtschaftsteuernde Fähigkeiten des Staates. Dieser unterscheidet zwischen konjunkturellen und strukturellen Defiziten. Hier wurde bei der Umsetzung der Schuldenbremse im Bundeshaushalt hinsichtlich der Größe des strukturellen Defizits gleich zu Beginn politisch "nachgebessert“. Der Finanzminister hat „die Ausgangslage kurzfristig verändert, man orientiert sich jetzt nicht mehr an dem Datenkranz, der bei Aufstellung des Haushalts 2010 vorlag, sondern an der aktuellen Entwicklung. Sonst hätte es den merkwürdigen Umstand gegeben, dass im kommenden Jahr die zulässige Verschuldung höher gewesen wäre als die nun für dieses Jahr zu erwartende Verschuldung"18.

Die Konjunkturkomponente des Defizits soll sich als Produkt aus der Produktionslücke und der Budgetsensitivität ergeben. Unter Produktionslücke wird die Unter- oder Überauslastung der gesamtwirtschaftlichen Produktionskapazitäten verstanden. De facto handelt es sich hier um eine Schätzung, da der Bezugsrahmen das laufende Haushaltsjahr ist. Dem Gesetzgeber waren die damit verbundenen Unsicherheiten wohl bewusst, denn er fordert, die entsprechenden volkswirtschaftlichen Verfahren in Übereinstimmung mit dem im Rahmen des Europäischen Stabilitäts- und Wachstumspakts angewandten Konjunkturbereinigungsverfahren regelmäßig zu überprüfen und fortzuentwickeln. Unter Budgetsensitivität wird ein Zahlenwert verstanden, der angibt, wie sich die Einnahmen und Ausgaben des

17 Gideon Rachman, Sweep Economists off their Throne, in: Financial Times vom 7. September 2010, S. 9.

18 Manfred Schäfers, a.a.O. (Fn. 4). 
Bundes bei einer Veränderung der gesamtwirtschaftlichen Aktivität verändern. Hier zeigt sich wieder die problematische Annahme, man könne im Zeitalter der Globalisierung solche Zahlenwerte für politische Entscheidungen zeitgerecht und exakt ermitteln oder gar im nationalen beziehungsweise für konjunkturbedingte Verschuldungsmöglichkeiten auch im Länder-Rahmen finden. ${ }^{19}$ Die OECD stellt hierzu fest: „Im Rahmen der neuen Haushaltsregel ist die Ermittlung der Position der Wirtschaft im Konjunkturzyklus von zentraler Bedeutung. Es ist allerdings schwierig, ein verlässliches Maß für den Konjunkturzyklus zu ermitteln, da sich das Produktionspotenzial nicht beobachten lässt und jede Messung der Lücke zwischen effektiver und potenzieller Produktion somit in hohem Maße ungewiss ist und häufig Gegenstand erheblicher Revisionen im Zeitverlauf wird. “20

Neben den Problemen der „Neutralität“ und des „keynesianischen Steuerungsglaubens“ ist als drittes Effizienzproblem das der wirksamen Sanktionen bei Verstößen gegen die neuen Haushaltsregeln zu nennen. Nach den Erfahrungen mit der Euro-Krise gehört Deutschland zu denjenigen Ländern, die eine Verschärfung der Sanktionsmöglichkeiten des europäischen Stabilitäts- und Wachstumspaktes fordern. Innerstaatlich wurden entsprechende Lehren vermieden. Mehr als eine Bitte zur Besserung kann der Stabilitätsrat an einen Defizitsünder nicht herantragen. Wie wirksam eine solche „Aufforderung“ sein kann, bleibt abzuwarten. Parteipolitik und Wahlkämpfe als entgegenwirkende Motive wird auch der Stabilitätsrat nicht in den Hintergrund drängen können. Geldstrafen hätten wohl angesichts der Hürden für eine Entscheidung im Stabilitätsrat ohnehin keine Chance, akzeptiert zu werden, zumal deren Sinn zur „Bestrafung“ hochverschuldeter Länder nicht recht einleuchtet.

Ein viertes möglicherweise auftretendes Effizienzproblem sind die Ausnahmeregelungen. Es bleibt zu hoffen, dass die Definition „außergewöhnlicher Notsituationen“ strikt gehandhabt wird und sich nicht in eine generelle Öffnungsklausel „weiterentwickelt“. Daneben droht auch eine Flucht in alternative Finanzierungsformen wie Public Private Partnerships oder das Ausweisen von Zahlungen an Unternehmen oder andere Institutionen als Darlehen oder Kapitalzuführungen, die formal außerhalb der regulären Haushalte stattfinden und deshalb bei der Berechnung von Defiziten keine Rolle spielen.

Fragen der föderalen Ordnung stellen sich im Kontext der neuen Haushaltsregeln auf mindestens zwei Ebenen, zum einen bei der Durchführung, zum anderen prinzipiell. Die deutsche Politikverflechtung ist wenig geeignet, eine Schuldenbremse wirksam durchzuführen. Die fehlende Haushaltsautonomie der Gliedstaaten in Verbindung mit der Gemeinschaftsfinanzierung wesentlicher Staatsaufgaben erlaubt weder, einen Rechtfertigungszwang für die Politik gegenüber den Wählern aufzubauen, beispielsweise durch die Möglichkeit von Steuererhöhungen wegen mangelnder Haushaltsdisziplin, noch den Ländern als Sanktionsmöglichkeit bei Fehlverhalten beispielsweise Mittel im Rahmen der Mischfinanzierungen zu entziehen. Neben den Wählern fallen auch die Finanzmärkte als externe Kontrollinstanzen aus, da sich diese einem einheitlichen Rating aller Länder und des Bundes gegenüber sehen. Solange die Länder sicher sein können, letztendlich vom Bund bezie-

19 Vgl. Uwe Berlit, Die Umsetzung der Schuldenbremse in den Ländern - erste Ansätze und Probleme, in: Martin Junkernheinrich / Stefan Korioth / Thomas Lenk / Henrik Scheller / Matthias Woisin (Hrsg.), Jahrbuch für öffentliche Finanzen 2010, Baden-Baden 2010, S. 311 - 342, S. 334 .

20 OECD, a.a.O. (Fn. 16), S. 69 f. 
hungsweise der Gemeinschaft der Länder herausgekauft zu werden, wird sich das LänderRating nicht entsprechend der Länderleistung ausdifferenzieren.

Die Politikverflechtung im deutschen Föderalismus spiegelt sich bei der Zusammensetzung des Stabilitätsrats wider. Angesichts der hohen Hürde für seine Beschlüsse ist gerade keine strenge Linie bei der Defizitkontrolle zu erwarten, eher der kleinste und damit politisch weniger schwierig durchzusetzende gemeinsame Nenner und im schlimmsten Falle "Zeitinkonsistenz", also eine erratisch sich verändernde Politik, die sich von ihren Ausgangsvoraussetzungen wegbewegt. ${ }^{21}$ Das Argument, die Haushaltsregeln verschafften „Verfahrenstransparenz", die zu einem erheblichen öffentlichen Druck auf eine Gebietskörperschaft bei Fehlverhalten führt, ist oberflächlich und nimmt weder die politische Präferenzbildung noch den Kontext der neuen Haushaltsregeln, nämlich die fortdauernde Politikverflechtung und das Desinteresse der Öffentlichkeit an den Mechanismen der Schuldenbremse wahr.

Festzuhalten ist auch: Die neue Verschuldungsregel nimmt den Landesparlamenten, die schon bisher auf der Einnahmenseite weitgehend einflusslos waren, weil der Bund die wesentlichen Steuergesetze verabschiedet (unter Mitwirkung des Bundesrates), nun auch weitestgehend ihre Autonomie auf der Ausgabenseite. Wo noch Spielräume sein mögen, wirkt der „goldene Zügel“ des Bundes zentralisierend. Da nun nur noch der Bund sich jährlich neu verschulden darf, bleibt zu hoffen, dass sich die Länder nicht dadurch Finanzspielräume beschaffen, dass sie ihre Kommunen knapper halten. Was aber, die Frage muss erlaubt sein, ist ein Landesparlament ohne die Möglichkeit, im Wesentlichen die eigenen Einnahmen und Ausgaben zu bestimmen, eigentlich noch wert? Zumal es zunächst nicht einmal gefragt wurde, ob es einer Schuldenbremse zustimmt oder diese in die Landesverfassung aufnehmen möchte. Ist die Schuldenbremse die letzte Stufe der Selbstaufgabe der Länder? Oder soll man mit Christian Waldhoff und Peter Dieterich optimistischer sein, wenn sie meinen: „Die Länder werden durch das neue Regime ,an die Kette gelegt', doch erweist sich diese als ausreichend lang, um eine Strangulation zu vermeiden." 22

21 Vgl. Friedrich Gröteke / Karsten Mause, Die deutsche „Schuldenbremse“: Ein wirksames Instrument zur Vermeidung eines Bailout?, in: Zeitschrift für Wirtschaftspolitik, 58. Jg. (2009), H. 3, S. $309-335$.

22 Christian Waldhoff/ Peter Dieterich, Die Föderalismusreform II - Instrument zur Bewältigung der staatlichen Finanzkrise oder verfassungsrechtliches Plazebo?, in: Zeitschrift für Gesetzgebung, 24. Jg. (2009), H. 2, S. 97 - 123, S. 116. 